\title{
The Application of Intelligent Materials in Intelligent Product Design Yuan Gao ${ }^{1}$, Wei Xiao ${ }^{2}$, and Mei Gu ${ }^{3}$ \\ ${ }^{1}$ Anhui Polytechnic University, Wuhu, Anhui Province, PRC, 241000. \\ ${ }^{2}$ Fuyang Normal University, Fuyang, Anhui Province, PRC 236041; \\ ${ }^{3}$ Anhui Polytechnic University, Wuhu, Anhui Province, PRC, 241000. \\ ${ }^{*}$ Corresponding author
}

Keywords: Intelligent Materials, Intelligent Products, Product Design, Application.

\begin{abstract}
The products of intelligent design are rapidly growing in popularity around the world and are having increasing impact on humans' life styles. The application of intelligent materials, the basis of intelligent products in intelligent product design, is a current research hot spot. This paper, based on the application of intelligent products, will start with the basic characteristics and classification of intelligent materials. The application of intelligent materials in intelligent product design will be discussed from four perspectives: interesting intelligence, intelligent processing, adaptive intelligence and intelligent communication.
\end{abstract}

\section{Introduction}

With the continuous progress of human society and the rapid development of computer technology and the Internet of things, the living standard of human society has been greatly improved. Simultaneously, people's requirements for product safety, health, comfort, convenience and other auxiliary functions are also rising exponentially. These rapid technological advances have enriched people's material and spiritual life considerably. In turn, the improvements in people's lives brought about by these new technologies is fueling ever increasing demand for enhanced product function and new product based upon the same intelligent platforms. Thus, many new and revolutionary smart products are emerging such as smart homes, smart medical products, smart cars and smart environmental, energy storing and energy saving products.

Intelligent material is at the core of the design and application of intelligent products. The accurate use of smart materials is not only essential for the realization of the function and appearance of intelligent products, but also affects the subsequent expansion and improvement of functions of intelligent products. At present, research on new materials is ongoing and newer materials and technologies are emerging. For a new product, materials and design are always complementary and both of them must play their due role in a particular product's creation and evolution especially smart products with advanced technological features. Intelligent material is the basis of intelligent product design and the design process is a means to achieve the function of intelligent materials; that's to say, smart material is the object of design and each design is ultimately required to be applied to the material. Therefore, it is of great significance to study the application of smart materials in intelligent product design.[1]

\section{Definition and Analysis of Basic Characteristics of Intelligent Materials}

Definition of intelligent materials. Intelligent materials refer to the materials that have the ability to perceive external stimuli, to judge and properly handle it and can implement itself. The idea of smart material originates from Bionics while its initial goal was to develop a "living" material that is similar to biological functions within a living organism. Therefore, smart materials must have three basic elements: perception, drive and control.

Specifically, smart materials should have the following properties. Firstly, a perceptive function. Intelligent materials are defined by their ability to realize self-adaptive sensing and detection of 
internal and external information. Some intelligent products also need intelligent materials to offer an effective feedback of the intensity of internal and external information. Secondly, a driving function. Intelligent materials need to perceive and respond to internal and external information. They need to have the ability to select and control responses in a prescribed manner. What's more, the materials should have a certain response sensitivity that can make appropriate and moderate response in a certain response time. Thirdly, an automatic recovery function. Intelligent materials must be programmed in a manner that they automatically return to their original state when the stimulus from the inside and outside disappears.

Analysis of basic characteristics of intelligent materials. Smart materials often have or have some of the following Intelligent functions and characteristics.[3]

(1) Sensor: This is the basic function of intelligent materials which are able to perceive and detect stimuli from the external environment or their own conditions. These stimuli include load, stress, strain, vibration, heat, light, electricity, magnetism, chemistry and nuclear radiation, etc.

(2) Feedback: Intelligent materials can compare the information of parameters of the input system with the output information through the materials themselves or the sensor network of system configuration. What's more, they can offer a feedback of the results to the system controller to carry out feedback control.

(3) Function of recognizing and accumulating information: Intelligent materials are able to identify all kinds of parameters acquired by a sensor network, even to classify and sort out information and output it according to the type of information. What's more, they can accumulate the collected parameters and save them to a certain time according to programmed requirements.

(4) Function of response: Intelligent materials can make real-time and dynamic responses according to changes in the outside or inside environment and take appropriate action as required.

(5) Self-diagnosis: Intelligent materials can analyze and compare the current state with the historical state of the system. The analysis and comparison of the information differences among them are beneficial to self-diagnosis of such problems as system faults and wrong judgment which allow the smart product to conduct automatic calibration under certain input conditions.

(6) Self-recovery: Intelligent materials cannot only conduct self-diagnosis of the working state, but also diagnose the problems in the operational process and conduct self-recovery of those problems. The materials realize a combination of self-diagnosis and self-recovery.

(7) Self-adjusting: Intelligent materials can adjust their structures and functions according to the continuous changing environment or the stimuli. The materials will adjust their own states and behaviors according to external changes to always keep the optimum condition to ensure the functional realization of the products.

\section{Application of Intelligent Materials in Product Design}

Nowadays, the variety of at materials that can be used for intelligent materials is growing, so intelligent material is at the core of a large number of methods to classify smart materials. Generally, according to the required function, materials can be divided into optical fiber, shape memory alloy, piezoelectric and electronic (magnetic) elastic material, etc. In terms of the sources, the materials can be divided into metallic intelligent materials, inorganic non-metallic intelligent materials and high polymer intelligent materials.[4] Metallic intelligent materials have emerged through the research and development of shape memory alloys as well as shape memory composites. Inorganic non-metallic intelligent material are quickly emerging in such areas as electro rheological fluids, piezoelectric ceramics, photo chromic and electro chromic materials. High polymer intelligent materials come in a wide range, for instance, polymer gel, intelligent polymer film, intelligent drug release system and intelligent polymer matrix composites.

Interesting Intelligence. Modeling, color, material and function are the basic elements of a product. Intelligent product, a commodity facing increasingly severe market competition environment, must be treated more strictly than other general industrial products in terms of its function of arousing interests. Under the support of intelligent material technology, the product's 
function of arousing consumer interest can be realized by rich shapes and colors, reasonable material application which can help users generate a richer experience during the process of use. Under these circumstances, it is not enough for designers to just focus on the interesting appearance of the product design. Simply relying on the beautiful appearance of the design is not a strong basis for ensuring a product's lasting competitiveness. In order to improve product experience with a reasonable collocation between design and function, the products, based on intelligent materials, must mainly focus on improved functionality. The goal of an intelligent product designer should be to create an enhanced 'fun' environment as the users are experiencing products. Currently, the widely-used smart phone is a good example. Designers realize a phone's basic functions through the built-in smart chip and a variety of smart materials with perceptive function, and, at the same time, they make it thinner, lighter and more convenient for people's daily use. In terms of the special functions of smart phones, designers are more inclined to the realization of mobile phones' self-adaptive functions such as automatic navigation, automatic search and intelligent replies, etc. What's more, the changeable shapes and colors of smart materials help add some four-dimensional features such as time, dynamics, and plot into the three-dimensional features such as length, width and height in original product design, which makes the products provide static experience feeling and also improves the dynamic fun in the process of stress changes caused by smart materials and design.

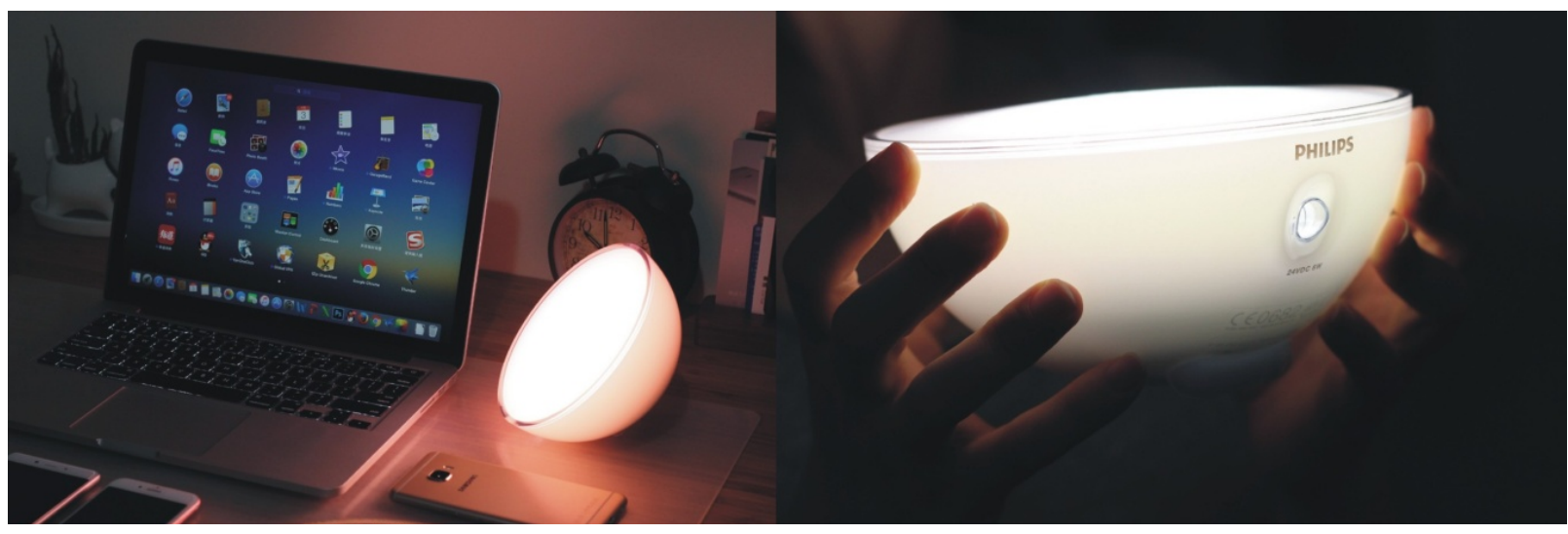

Figure1.smart light Hue Go of Philips

The picture above is a smart light product called 'Hue Go' from Philips which, through the use of smart materials, supports 16 million colors all controlled by the Hue App with the help of wireless communication technology called Zigbee. The color, brightness and switch time of the lamp can be adjusted both manually or through the APP. It can not only provide a variety of photo effects, but also reflect interactive information from a smart phone such as change colors according to the phone's music rhythm or screen content. It is an extremely interesting experience that when the user holds a mobile phone to play games, the GO changes color with the rhythm of the game, or echoes the game with light. However, this experience would not exist without the intelligent materials and intelligent product design that can respond to cell phones.

Intelligent processing. As humanized demand is an important aspect of today's product design, the intelligent product is able to meet humanized demand. In modern time, with the increasingly faster rhythm of life, people need to deal with many complicated affairs every day. The complexity of contemporary life consumes ever greater amounts of peoples' time and energy. Consequently, there is a rising demand for products that can lessen peoples' burdens of dealing with their daily affairs. Smart products have just this function, thus, they are eagerly sought after by people. However, there are deep reasons for the rapid expansion of the smart product market. Smart materials can be programmed with an ability similar to independent thinking and judgment-making which endow intelligent products with more powerful functions. They can be programmed to take the initiative to complete a requested action rather than passively waiting for a person's response. The intelligence of this process depends not only on the high degree of product information and 
control technology, but also depends more on the stress and drive of the smart material itself. The stress and drive of this dynamic change endow the product with the ability to think intelligently and deal with emergent situations according to the judgment based on thought. Highly intelligent products are also capable of operating automatically during function transformation or switch operation, completing the required mission without external orders.

Intelligence of product-handling capabilities brought about by smart materials has completely changed human's operating habits of non-intelligent products and provides users with a heightened user experience. Now, many intelligent products have improved human-computer interaction function. Powerful chip and the application programs give full play to the functions of smart materials. After the acquisition of external commands, smart products can take the initiative to achieve more complex action, which is based on the support of powerful smart materials.
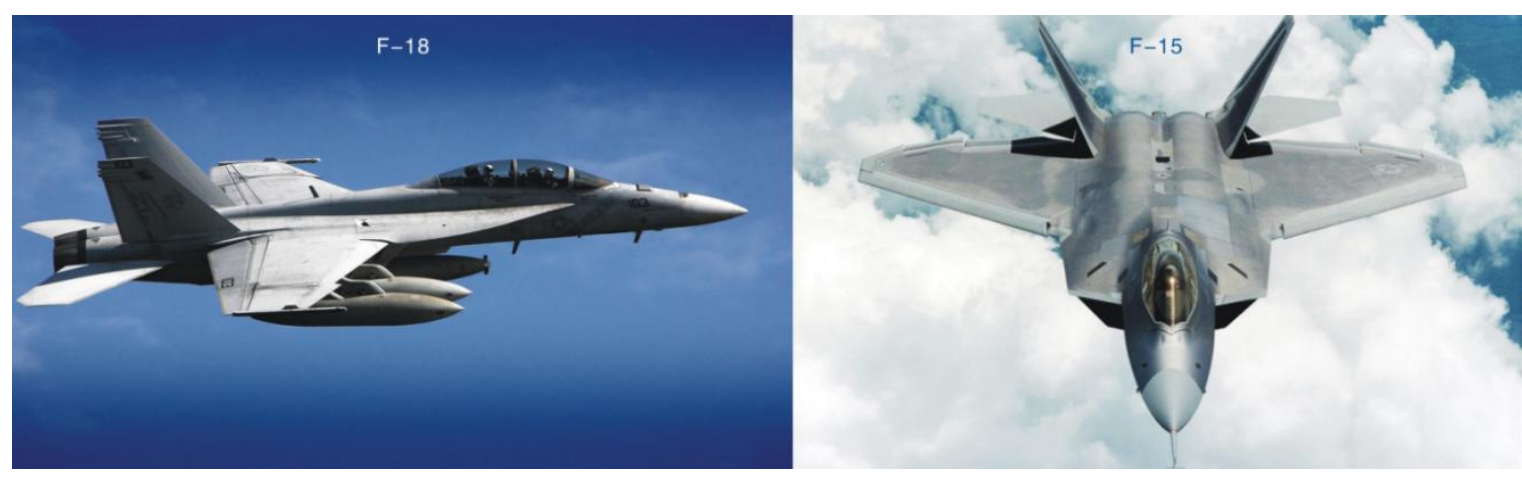

Figure 2.aircraft with intelligent skin

For example, the smart covering on these pictured fighter aircraft, uses super light material and optical fiber as intelligent sensors allowing the installation of advanced micro processing control systems on the surface of the aircraft, turning a traditional aircraft skin into an airborne communication antenna and synthetic aperture radar. The skin can detect and process all kinds of information and threats from the air, significantly reducing the amount of machine space whilst significantly increasing weapons and fuel load. Overall, the utilization of intelligent skin greatly improves the effectiveness of aircraft communication and early warning information processing as well as it's stealth effect and flight and combat performance.

Adaptive intelligence. Adaptability can solve all problems associated with the human body during product design such as the parameters of the morphological characteristics of the human body, human's perception characteristics and human's response characteristics. Therefore, the problem of how to adapt product design to the physiological and psychological characteristics of human beings can be readily solved. The adaptability of the product may include the adaptability of man-machine size, the adaptability of the user's operational preferences and the adaptability of the smart tool to the user's psychological preferences.

In the design of intelligent products, in addition to the careful selection of smart materials, the designers also need to tailor product design and adaptability parameters to market requirements. Information guiding appropriate adaptations comes from a wide range of market research and the accumulative collection of customer experience and advice. The success of any smart product largely depends upon the overall quality and flexibility of the adaptive intelligence and customers response and approval of its flexibility and adaptability. For example, building materials made of smart materials have the ability to automatically perceive environmental components and can automatically secrete anti-corrosive substances to protect the strength of the building structure when the environmental $\mathrm{pH}$ increases. Such products will definitely be welcomed because the use of intelligent materials improves product performance and quality and saves significant maintenance costs and energy.

Intelligent Communication. The work efficiency of man-machine 'smart' systems depends on its whole operation rather than one aspect. The crux of a highly effective man-machine 'smart' system is enhanced communication between man and machine. 
The intelligent communication of intelligent material is reflected in the following aspects.

(1) Intelligent materials have the capacity to make make suggestive communication. In other words, when the user needs to enter external instructions, the smart materials are programmed to offer the user effective operational tips. Therefore smart materials must be programmed with accurate action information in a bid to provide the user with clear and precise indicative information. Suggestive tips can be manifested by some intuitive conversation interfaces for example through the use of attention-grabbing shapes and colors.

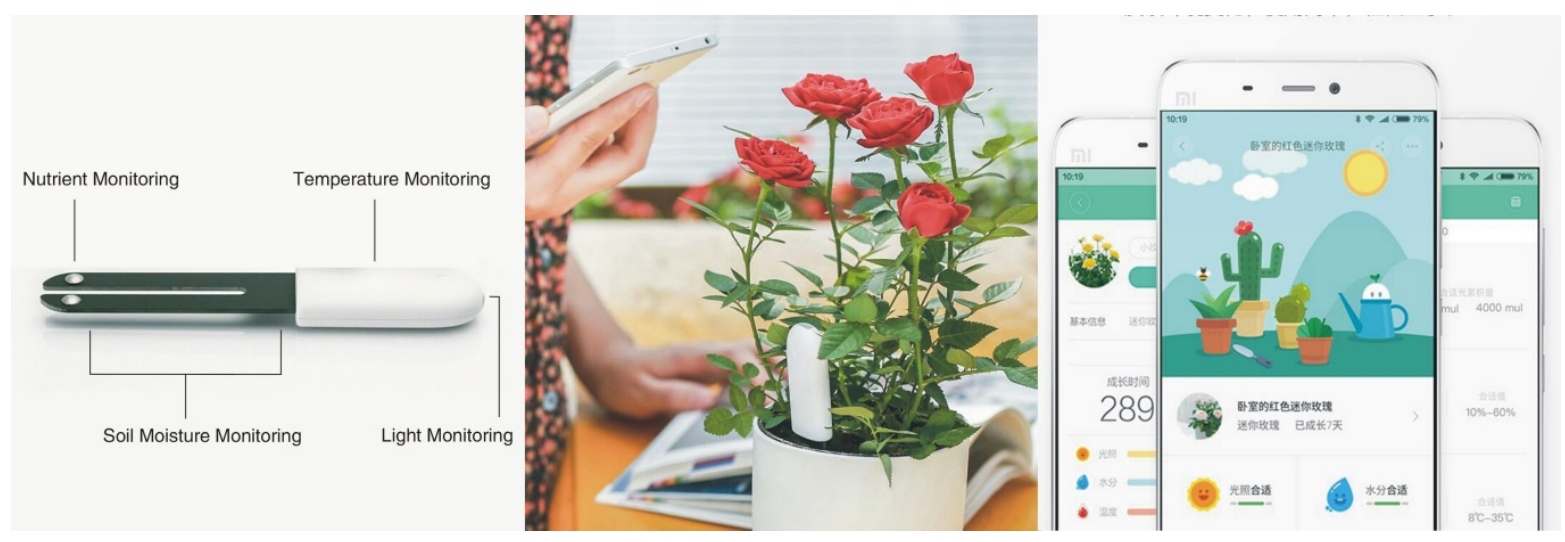

Figure 3.intelligent diagnostic instrument for raising flowers

As an example, we cite the intelligent diagnostic instrument for flower cultivation created by Xiaomi, a famous Chinese brand. With the help of intelligent material that can be used to sense the soil $\mathrm{pH}$ and humidity, relevant data from soil can be obtained and then transferred to an APP by wireless system. This APP will calculate the data, compare it with the relevant plant data in the database and then draw a conclusion which can instruct the user on the improved cultivation of the plants.

With the development of intelligent materials, the intelligent products even have the possibility of conducting man-machine communication through such interactive modes as smell and touch.

(2)Intelligent materials can be programmed to facilitate interactive communication. The value of communication comes from mutual understanding and cooperation rather than operating or providing information unilaterally. The interaction between products and users can be seen everywhere in industrial products and the effectiveness and speed of intelligent materials in this interaction are very evident. A closer and effective interaction between intelligent products and users is able to enhance user's command ability and offers timely feedback to smart materials to make the corresponding action. The objective is the realization of a smooth and seamless operation between man and machine.

For example, the intelligent materials created for the manufacture of artificial skin can sense the slightest changes of temperature within heat flux and the size of various stresses whilst demonstrating good spatial resolution. Its integration and acceptance by the organic body benefits from its continuous communication with other human organs. This multifold communication assists it in obtaining compatible information and thus its seamless integration by the organic body concerned.

\section{Conclusions}

The rapid development of smart materials brings us novel experience and superior product performance. Thus, the era of the great popularization and application of intelligent material is developing exponentially. In order to design more and better intelligent products, it is necessary to fully explore and develop the characteristics of the intelligent material as well as work out an application mode through the combination of creativity and innovation. Clearly, the key to rapid advances in this field lies in ensuring a seamless blending of all four of the key characteristics of 
successful intelligent design outlined in this paper viz, interesting intelligence, intelligent processing, adaptive intelligence and intelligent communication.

\section{References}

[1] Chen Li, Intelligent polymer materials, Chemical Industry Press, Beijing, 2005.

[2]WEIZG, SANDST ROMR, Review for Shape-Memory Material and Hybrid Composites for Smart Systems, Journal of Materials Science, 33 (1998), pp. 3743 -3762.

[3] ChuncaiZhang, JiacanSu, Shape memory materials, Second Military Medical University press, Shanghai, 2003, pp. 1-10.

[4]TianwenLi, HongshengLiu, Research and application of color changing material, Modern chemical industry, 2 (2004) 62-70.

[5] LianMo, Research on product design based on life form,30 (2013) 127-128.

[6] JinshengZhang, HongyuGong et al, Application of functional materials, Journal of Shandong University (Engineering Science Edition), 32 (2002) 295-298. 